3 Kosaka K, Matsushita M, Oyanagi S, Mehraein P. A clinicopathological study of the "Lewy body disease". Psychiatria et Neurologia faponica 1980;82:292-311.

4 Edwards LL, Pfeiffer RQ, Quigley EMM, et al. Gastrointestinal symptoms in Parkinson's disease. Mov Disord 1991;6:151-6.

5 Edwards dwards LL, Quigley EMM, Harned RK, Hofman R, Pfeiffer RF. Characterisation of disease. Am $\mathcal{f}$ Gastroenterol 1994;89:1525.

Local IgG production in aqueous humour in patients with idiopathic uveitis compared with MRI findings

We have previously reported an increased concentration of antibody to measles in the aqueous humour of patients with multiple sclerosis related uveitis. ${ }^{1}$ We further found a high frequency of asymptomatic multiple sclerosis-like lesions on MRI of the brain of patients with idiopathic uveitis. ${ }^{2}$ There was a significant association between MRI lesions and high aqueous humour and blood concentrations of antimeasles antibodies. $^{3}$

The local production of oligoclonal IgG in the CNF has long been considered as a pathognomonic characteristic of multiple sclerosis, and also a good biological aid to its diagnosis. ${ }^{4}$ Oligoclonal production of IgG has also been suspected in the aqueous humour of patients with multiple sclerosis, on the basis of the ratio between albumin and IgG contents in both aqueous humour and serum. ${ }^{5}$

This study was aimed at determining if the presence of white matter MRI lesions in patients with uveitis was linked to an increased content of IgG in the aqueous humour, and if this increase could be explained by the high concentration of antimeasles antibodies in the aqueous humour.

Sixty eight patients with idiopathic uveitis (23 males, 45 females, aged 15-49), all free of neurological symptoms, diabetes, and blood pressure problems, were submitted to both a brain MRI examination and a sampling of blood and aqueous humour. All patients gave informed consent to their participation in this study.

The presence of white matter lesions on MRI was determined in each patient by three independent radiologists according to Paty's multiple sclerosis diagnosis scale. ${ }^{6}$

Samples of aqueous humour and blood were assayed for albumin and total IgG by laser nephelometry and measles virus, herpes simplex virus (HSV), varicella zoster virus (VZV), and cytomegalovirus (CMV) specific IgG by enzyme linked immunosorbent assay (ELISA). The following coefficients were calculated ${ }^{5}$ : albumin quotient $=$ aqueous humour albumin/serum albumin; IgG quotient = aqueous humour IgG/serum
IgG; IgG index = IgG quotient/albumin quotient.

The values of these three coefficients as well as optical densities in aqueous humour for each of the four virus assays were compared in patients with lesions on MRI $(\mathrm{MRI}+)$ and those without (MRI-) by Wilcoxon's test. Partial Spearman correlations were calculated to assess if the relation between the local IgG production coefficients and MRI was related to a specific viral expression.

The results showed an association between the presence of brain lesions and high ocular IgG production as represented by the IgG quotient $(P=0.002$; table). Albumin quotient did not differ significantly between the groups and the association was due to differences in the values of the IgG quotient $(P<0.05)$.

Moreover the measles optical density in aqueous humour of MRI + patients was significantly higher than that of MRIpatients. $(P<0.0001)$. We did not find a difference in VZV, HSV, or CMV optical densities between the groups. The values of the measles optical density in aqueous humour were significantly correlated with both IgG quotient $(P<0.01)$ and IgG index $(P=0 \cdot 04)$. Finally, when adjusting for the effect of measles optical density in aqueous humour, the partial correlation between IgG index and MRI remained significant $(P<0.05)$.

The well documented relation between multiple sclerosis and uveitis is difficult to evaluate from a clinical point of view. Asymptomatic forms of ocular inflammation have been variously described in multiple sclerosis. The local production of $\mathrm{IgG}$ in the CSF of patients with multiple sclerosis is also well documented. ${ }^{4}$ White matter lesions on MRI of the brain are present in various inflammatory diseases, but specially multiple sclerosis.

The aim of this study was to check if the IgG concentration was increased in the aqueous humour of patients with idiopathic uveitis. The albumin quotient measures the modifications of the blood ocular barriers and by comparing both albumin and IgG quotient, the local production of IgG (IgG index) can be evaluated. The association between a high IgG index in aqueous humour and the presence of white matter lesions in patients with idiopathic uveitis and without neurological symptoms might be a phenomenon similar to the local increase in the IgG index in the CSF of patients with multiple sclerosis.

Our study is based on a quantitative measure of IgG production that is less sensitive than the qualitative measure of oligoclonal IgG. We think, however, that the large number of patients studied compensates for the lower sensitivity of our measures, compared with studies based on qualitative measures that involve a smaller number of patients.

The common presence of measles anti- bodies in the CSF of patients with multiple sclerosis is well known. ${ }^{7}$ After adjusting for the values of measles optic density in aqueous humour, the correlation between IgG index and MRI remained significant. This indicates that measles optical density in aqueous humour and IgG index are positively linked with MRI but the link between IgG index and MRI lesions is only partially explained by the link between measles optical density in aqueous humour and MRI. If measles has a role in the origin of multiple sclerosis and multiple sclerosis related uveitis, it may not be the only contributing factor.

We acknowledge the Association pour la Recherche sur le Sclérose en Plaques (ARSEP).

SERGE KOSCIELNY Département de statistiques médicales, Institut Gustave Roussy, Villejuif, France PHILIPPE THULLIEZ Laboratoire de la toxoplasmose, Institut de Puériculture,

EMMANUEL-AIAIN CABANIS MARIE-THERĖSE IBA-ZIZEN Service de Neuroradiologie, CHNO des quinze-vingts, Paris, France ELISABETH DUSSAIX Laboratoire de Virologie, CHU Bicêtre, Le Kremlin Bicêtre, France ETIENNE BLOCH-MICHEL CLAUDINE TRICHET LAURENT HELLEBOID Unité d'immunologie oculaire,
CHU Bicêtre,

Le Kremlin Bicêtre, France

Correspondence to: Dr E Bloch-Michel, Unité d'immunopathologie oculaire CHU de Bicêtre, Faculté de médecine Paris Sud, Le Kremlin Bicêtre, France.

1 Bloch-Michel E, Helleboid L, Hill C, Koscielny S, Dussaix E. Measles virus antibody in aqueous humour of patients with uveitis associated with multiple sclerosis. Lancet 1992;339:750-1.

2 Bloch-Michel E, Iba-Zizen MT, Koscielny S, et al. MRI and idiopathic uveitis in: Dernouchamps JP, Verougstraete C, Cadvances in uveitis. Amsterdam: Kugler, advances in

3 Helleboid L, Dussaix E, Tounzi Y, Thulliez Koscielny S, Bloch-Michel E. The role of neurotropic viruses in the etiology of uveitis. In: Dernouchamps JP, Verougstraete $\mathrm{C}$, Caspers-Velu L, Tassignon MS, eds. Recent advances in uveitis. Amsterdam: Kugler, 1993:253-6

4 Waksman BH. Multiple sclerosis. Curr Opin Immunol. 1989:1:733-9.

5 Quentin CD, Reiber H. Intraocular immunoglobulin $G$ production in patients with perivasculitis of the retina and multiple sclerosis. In: Secchi AC, Fregona IA, eds. sclesis. In. pathology of the eye. Milano: Masson, pathology of

6 Paty DW, Oger JJF, Kastrokoff LF et al. MRI in the diagnosis of MS. Neurology 1988;38:185.

7 Felgenhauer K, Schadlich HJ, Ackerman R. Cerebrospinal fluid virus antibodies: a diagnosis indicator for multiple sclerosis. f Neurol Sci 1985;71:291-9.

\section{Central nervous system demyelination after vaccination against hepatitis $B$ and HLA haplotype}

Although very unusual, acute CNS complications may occur after vaccination against hepatitis B. ${ }^{1}$ We report a patient with evidence of severe demyelination in the CNS after hepatitis B vaccination. 
A 35 year old man, with no medical history and no alcohol, tobacco, or drug misuse, presented in December 1992 because of weakness and tingling in both legs, fatigue, and recent diplopia. The symptoms began in June 1992, two weeks after a third injection of plasma derived hepatitis B vaccine (Hevac B, Pasteur), and gradually worsened. The patient reported the same symptoms, that lasted a week, after a second injection in May 1992, and he had experienced nausea and unsteadiness of 10 days duration, three weeks after the first injection of a recombinant hepatitis B vaccine (GenHevac B, Pasteur) in March 1992. These symptoms were at that time misinterpreted as digestive by the general physician and no reliable information on the neurological examination could be obtained.

Examination showed a Romberg positive sign, spastic paraplegia, right sided hyperreflexia, and Babinski's sign, without any evidence of impaired sensation.

Hepatitis B serology was positive for antiHbS antibodies (34 UI). Cerebrospinal fluid showed a raised total protein (82 $\mathrm{mg} / 100 \mathrm{ml})$, with 9 blood cells $/ \mathrm{mm}^{3}(90 \%$ lymphocytes), and no evidence of intrathecal immunoglobulin synthesis or oligoclonal bands. Blood cultures and tests for syphilis, Lyme disease, toxoplasmosis, and HIV were all negative. Electroencephalography was normal. Nerve conduction studies showed a diffuse sensory neuropathy of both arms and legs. Visual evoked potentials showed a bilateral delay; somatosensory potentials of the tibial nerve and auditory potentials were abnormal. T2 weighted MRI showed multiple high signals in the brain white matter. Blood HLA typing showed the B7, DR2 haplotype.

The patient was treated with adrenocorticotrophin for one month with a good clinical response, and there was no relapse during a two year follow up. In September 1994 , the patient returned to a full time job. Physical examination showed no more gait disturbance, but a right sided pyramidal syndrome was still present. Repeat MRI showed unchanged multiple signals in the white matter.

Vaccination against B hepatitis is usually safe and efficient. ${ }^{2}$ In 1991, however, Herroelen et al described two patients who developed demyelination in the CNS after recombinant $B$ hepatitis vaccination. ${ }^{3}$ Both patients had the B7, DR2 HLA haplotype, which is associated with multiple sclerosis, and indeed one patient had a history of multiple sclerosis. Despite this HLA haplotype, in our patient the absence of a medical history, of intrathecal immunoglobulin synthesis, the presence of a diffuse sensory neuropathy, and the temporal association with vaccination argue against the diagnosis of multiple sclerosis. Nevertheless, it is difficult to establish whether neurological symptoms are directly and only due to vaccination, or if the vaccine simply triggers the onset or the relapse of an underlying multiple sclerosis. The findings of the B7, DR2 haplotype in these three cases of severe CNS demyelination might be trivial, indicating underlying multiple sclerosis, but might also suggest a pivotal role of this haplotype, possibly through special antigen presentation. The immune response might then induce non-specific lesions of CNS demyelination, with clinical and radiological expression similar to those in multiple scle- rosis. This finding, in addition to previous reports, suggests that vaccination against hepatitis B could potentially induce CNS complications in patients with the HLA B7, of multiple sclerosis.

\section{G KAPLANSKI F RETORNAZ J M DURAND J SOUBEYRAND Service de Medecine Interne, Service de Medecine Interne, Hopital Sainte-Marguerite, 13009 Marseille, France}

1 Shaw FE, Graham DJ, Guess HA, et al. Postmarketing surveillance for neurologic adverse events reported after hepatitis B years. $A m$ F Epidemiol 1988;127:337-52

2 Gardner $\mathrm{P}$, Schaffner W. Immunization of adults. N Engl F Med 1993;238:1252-8.

3 Herroelen L, De Keyser J, Ebinger G. Central-nervous-system demyelination after immunisation with recombinant hepatitis B vaccine. Lancet 1991;338:1174-5.

\section{A case of progressive subcortical gliosis associated with deposition of abnormal prion protein (PrP)}

In 1967 Neumann and Cohn used the term of progressive subcortical gliosis to describe four patients presenting with a dementing neurological illness. ${ }^{1}$ The principal histological finding in all four cases was pronounced gliosis of the white matter, basal ganglia, thalamus, brain stem, and ventral horns of the spinal cord, in the absence of diagnostic hallmarks of either Alzheimer's disease or Pick's disease. Although up to now only a small number of cases have been described, progressive subcortical gliosis, synonymous with Pick's disease type II, has been accepted by some of the standard neuropathological textbooks. ${ }^{2}$

In this fournal a case of progressive subcortical gliosis was reported from our institution in $1989 .{ }^{3}$ In summary, a 58 year old woman experienced difficulty in focusing and reading for three years before presenting with a general deterioration in intellectual function, including a deficit of short term memory. She was also found to have an akinetic rigid extrapyramidal syndrome and oculomotor signs usually associated DR2 haplotype, with or without a history vaccination. Experience of the first thre

with the Steele-Richardson-Olszewski syndrome. Neuropathological examination of the brain showed "atypical features", which included pronounced astrocytosis in some cerebral cortical areas and subcortical structures such as white matter, caudate, putamen, and globus pallidus as well as Ammon's horn. Several brain stem structures and the cerebellum were similarly affected. There was a complete absence of cortical or subcortical neurofibrillary tangles and Pick bodies.

Recently, positive immunochemical findings suggestive of prion disease were detected in cases of the familial form of progressive subcortical gliosis. ${ }^{4}$ This gave us the impetus to review our case and apply the $1 \mathrm{~A} 8$ anti-PrP antiserum (courtesy of $\mathrm{Dr}$ C F Farquar) with formic acid pretreatment, which showed a striking positive reaction in the neuropil of the granular cell layer of the cerebellum. The PrP positive deposits were either rather diffuse or small, compact, and plaque like (figure). Immunocytochemistry with an anti- $\beta / A 4$ antibody (Dako Ltd) was also carried out, but no immunoreactivity was detected. Appropriate negative controls were used by omitting the primary antibody, and these showed no staining. In association with immunostains for $\operatorname{PrP}$ adjacent sections stained with Congo red and thioflavin $\mathrm{T}$ for amyloid were also studied, but no birefringent or fluorescent structures, suggestive of amyloid, were seen.

The immunocytochemical findings in this case have important implications for the classification of progressive subcortical gliosis and suggest that at least some forms of the condition are part of the range of human prion disorders rather than a subtype of Pick's disease in which pathological PrP does not occur. ${ }^{5}$ These results also emphasise the value of applying modern immunocytochemical and molecular biological techniques to cases with a presenile dementing illness, in which specific histological markers of known neurodegenerative diseases are absent.

$$
\begin{array}{r}
\text { T REVESZ } \\
\text { S E DANIEL } \\
\text { A J LEES } \\
\text { R G WILL } \\
\text { Department of Neuropathology, } \\
\text { Institute of Neurology, } \\
\text { Queen Square, } \\
\text { London WC1N } 3 B G, U K
\end{array}
$$

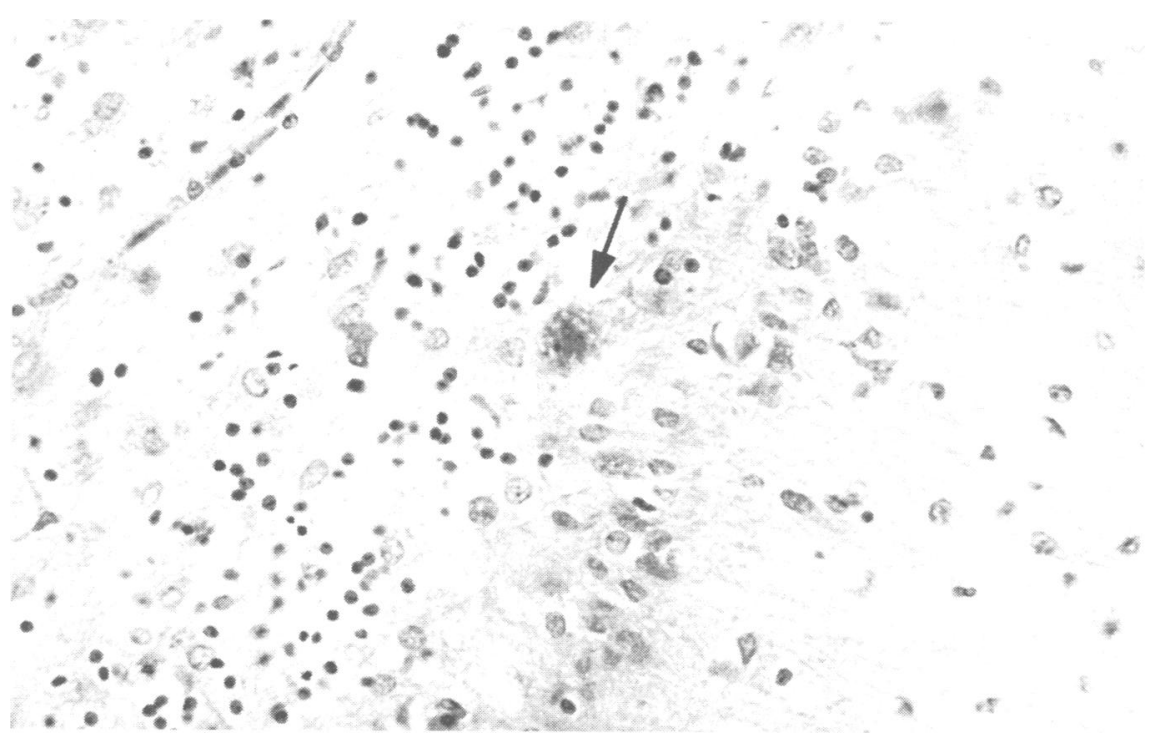

Small PrP positive, plaque like deposit in cerebellar cortex (arrow); PrP immunostain $\times 400$. 